\title{
Born to survive: how cancer cells resist CAR T cell therapy
}

\author{
Jean Lemoine ${ }^{1}$, Marco Ruella ${ }^{2}$ and Roch Houot ${ }^{3 *}$
}

\begin{abstract}
Although chimeric antigen receptor T cells demonstrated remarkable efficacy in patients with chemo-resistant hematologic malignancies, a significant portion still resist or relapse. This immune evasion may be due to CART cells dysfunction, a hostile tumor microenvironment, or resistant cancer cells. Here, we review the intrinsic resistance mechanisms of cancer cells to CART cell therapy and potential strategies to circumvent them.
\end{abstract}

Keywords: Immunotherapy, Lymphoma, Leukemia, Myeloma, Therapy

\section{Introduction}

In the last few years, chimeric antigen receptor (CAR) $\mathrm{T}$ cell therapy has emerged as a novel therapy for the treatment of B cells malignancies. Remarkably, CAR T cells can rescue patients who have failed multiple lines of therapies. Thus far, CAR T cells have been approved by the food and drug administration (FDA) for the treatment of $B$ cell acute lymphoblastic leukemia (B-ALL), diffuse large B cell lymphoma (DLBCL), mantle cell lymphoma (MCL), follicular lymphoma (FL) and multiple myeloma (MM). Despite this progress, a significant portion of patients still experience primary or secondary resistance to this treatment [1]. Resistance mechanisms to CAR $\mathrm{T}$ cell immunotherapy can involve the CAR T cells, the tumor microenvironment, or the cancer cells (Table 1) [2]. The current review focuses on cancer cells' intrinsic mechanisms of resistance to CAR T cells therapy, which include loss of the target antigen (Ag), expression of inhibitory receptors, lack of costimulatory ligands, and resistance to immune killing (Figs. 1 and 2). For each of these resistance mechanisms, we also discuss potential strategies which are envisioned to circumvent them (Table 2).

\footnotetext{
*Correspondence: roch.houot@chu-rennes.fr

${ }^{3}$ Department of Hematology, CHU de Rennes, Université de Rennes, INSERM U1236, 2 rue Henri Le Guilloux, 35033 Rennes Cedex 9, France Full list of author information is available at the end of the article
}

\section{Loss of target-Ag}

Thus far, loss of target-Ag has been the most widely studied mechanism by which cancer cells may resist or escape CAR T cell therapy (Fig. 1) [3-5]. Relapses with a target-Ag-negative clone may occur by immune-editing resulting in the selection of a pre-existing Ag-negative subclone, or possibly by acquired loss of the target-Ag that was initially expressed by the tumor cells.

\section{Incidence}

The incidence of Ag-loss after CAR T cell therapy may vary across histology. In B-ALL, CD19-loss accounts for approximately $7-25 \%$ of relapses after CAR T cells [6-10]. CD19-negative relapses seem to be associated with high tumor burden at the time of lymphodepletion [11]. In DLBCL, approximately a third of relapses exhibit CD19 loss or downregulation [12-18]. Importantly, CD19-negative or -low leukemia or lymphoma cells retain expression of other $B$ cell markers such as CD22 for B-ALL, CD20 and CD79a for lymphoma [18]. In MCL, Wang and colleagues reported 14 (23\%) relapses among the 60 patients in the primary efficacy analysis of the ZUMA-2 trial, of which 1 (7\%) had undetectable CD19 at relapse [19]. In FL, all 13 patients with evaluable tumor biopsies at progression in the ZUMA-5 trial had detectable CD19 [20]. In MM patients treated with CAR $T$ cells against $B$ cell maturation antigen (BCMA),

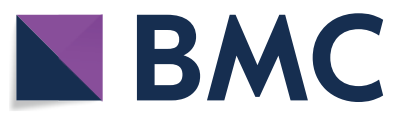

(c) The Author(s) 2021. Open Access This article is licensed under a Creative Commons Attribution 4.0 International License, which permits use, sharing, adaptation, distribution and reproduction in any medium or format, as long as you give appropriate credit to the original author(s) and the source, provide a link to the Creative Commons licence, and indicate if changes were made. The images or other third party material in this article are included in the article's Creative Commons licence, unless indicated otherwise in a credit line to the material. If material is not included in the article's Creative Commons licence and your intended use is not permitted by statutory regulation or exceeds the permitted use, you will need to obtain permission directly from the copyright holder. To view a copy of this licence, visit http://creativecommons.org/licenses/by/4.0/. The Creative Commons Public Domain Dedication waiver (http://creativeco mmons.org/publicdomain/zero/1.0/) applies to the data made available in this article, unless otherwise stated in a credit line to the data. 
Table 1 Mechanisms of resistance to CART cell therapy

\begin{tabular}{|c|c|c|}
\hline & Mechanisms of resistance & References \\
\hline CART cells & $\begin{array}{l}\text { Lack of expansion } \\
\text { Lack of persistence } \\
\text { Defective effector function (exhaustion) }\end{array}$ & $\begin{array}{l}{[7,8,94]} \\
{[7,8,94]} \\
{[95,96]}\end{array}$ \\
\hline Tumor microenvironment & $\begin{array}{l}\text { Impaired trafficking } \\
\text { Metabolism/Hypoxia } \\
\text { Immune suppression: } \\
\text { Immunosuppressive cells (stroma, myeloid cells, regulatory T cells) } \\
\text { Immunosuppressive cytokines (TGF-b, IL-10, IL-35) }\end{array}$ & $\begin{array}{l}{[97-101]} \\
{[102-104]} \\
{[105,106]}\end{array}$ \\
\hline Tumor cells & $\begin{array}{l}\text { Loss of target antigen } \\
\text { Expression of inhibitory ligands (PD-L1 expression) } \\
\text { Lack of costimulatory ligands (CD58 loss) } \\
\text { Resistance to immune killing }\end{array}$ & $\begin{array}{l}{[3-10,12-22]} \\
{[59-63]} \\
{[75-77]} \\
{[78-80]}\end{array}$ \\
\hline
\end{tabular}

1 (8\%) out of 12 treated patients exhibited biopsy-proven BCMA-loss at relapse, 8 patients remained BCMA-positive, and 3 were not evaluable for BCMA expression (no biopsy at relapse/progression) [21]. In another cohort, of 18 subjects with evaluable serial BCMA expression after BCMA CAR T cells infusion, 12 (67\%) had a decline in BCMA intensity on myeloma cells, including 4 out of 9 non-responders [22].

\section{Mechanisms}

Several mechanisms have been reported to explain Agnegative relapses, including selection of pre-existing Ag-negative tumor cells, mutation or splicing-variation, altered maturation/trafficking affecting target-Ag expression, epitope masking, and lineage switch/transdifferentiation (Fig. 1). Most data regarding the mechanisms of Ag-loss come from the clinical experience of CD19 CAR $\mathrm{T}$ cells in pediatric B-ALL. Flow cytometry analysis of 628 cases of relapsed or refractory (R/R) B-ALL from the Children's Hospital of Philadelphia (CHOP) revealed that, before any treatment, about $17 \%$ of cases had more than $1 \%$ of CD19-negative tumor cells. Moreover, $7 \%$ of patients had a decreased expression of CD19, and about a quarter had low-normal CD19 expression [23]. Thus, a significant number of patients have pre-existing tumor cells with absent or low membrane expression of targetAg which can be selected upon immune pressure from CAR T cells. Such CAR T cell-mediated immunoediting shapes the initial tumor heterogeneity favoring the emergence of target-Ag-negative relapses [24]. Furthermore, prior exposure to the CD19-directed, bispecific $\mathrm{T}$ cell engager (BiTE), blinatumomab was shown to be associated with a significantly higher rate of CD19-negative relapses after CAR T cell therapy [25].

\section{Mutations}

Flow cytometry analysis of 17 samples from pediatric and young-adult patients with R/R B-ALL showed that 12 patients had CD19-negative disease [7]. In all 12 patients, mutations in the CD19 domain were identified. These mutations were responsible for a truncated protein with a nonfunctional or absent transmembrane domain [26]. Such mutations affecting CD19 were also reported in the context of refractory DLBCL treated with CD19 CAR T cells [27] (Fig. 1B).

\section{Alternative splicing}

Sotillo and colleagues identified alternative splicing as a mechanism of Ag-loss by comparing CD19-negative samples at relapse with CD19-positive samples before CAR-T treatment from the same patients [28]. They found several CD19 splice variants expressed by B-ALL, especially affecting exon-2, resulting in the loss of the extracellular epitope of CD19 which is recognized by the CAR T cells. In other cases, alternative splicing resulted in lack of transmembrane domain precluding CD19 expression on the cell surface. Recent data reported the existence of CD19 isoforms at diagnosis which lack the CD19 epitope recognized by CAR $\mathrm{T}$ cells. This subclone may evolve as a dominant clone during CAR therapy and promote Ag-negative relapses [29] (Fig. 1B).

\section{Defect in Ag-processing}

Defect in maturation and trafficking of CD19 has been reported as a cause of resistance to CD19/CD3 BiTE, blinatumomab [30]. CD81 is a chaperone protein that regulates CD19 protein maturation and trafficking from the Golgi to the cell surface. In a patient, post-transcriptional regulation was responsible for CD81 loss, thus precluding CD19 processing and maturation in the Golgi. This alteration resulted in Ag-negative relapse 19 months after completion of blinatumomab treatment for B-ALL. This mechanism of resistance, reported with BiTE, may also be shared with CAR T cells, although not yet described (Fig. 1C). 

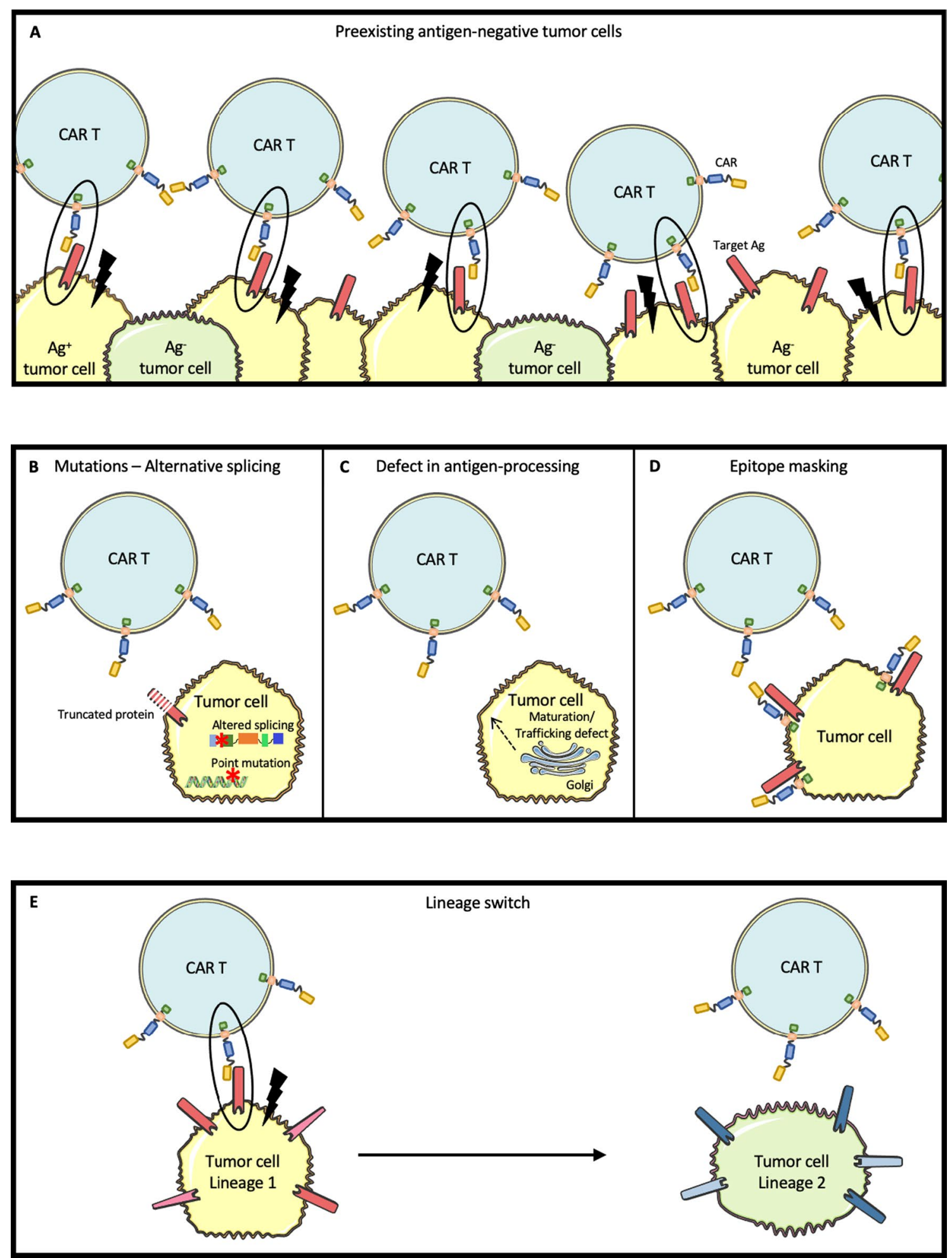

Fig. 1 Mechanisms responsible for loss of target antigen, conferring resistance to CART cell therapy. A Due to tumor heterogeneity before any treatment, pre-existing antigen-negative tumor cells may be responsible for resistance to CART cell therapy. B Point mutations or altered alternative splicing may lead to a truncated target antigen that can no longer be recognized by CART cells. C Defect in target antigen maturation and trafficking due to lack of appropriate chaperon proteins may be responsible for target antigen membrane expression loss. D Exceptionally, a tumor cell may be transfected with the CAR vector leading to an epitope masking by the CAR itself and hiding the target antigen from the CART cells. $\mathbf{E}$ Lineage switch may be responsible for a complete phenotypic markers remodeling including loss of the target antigen 


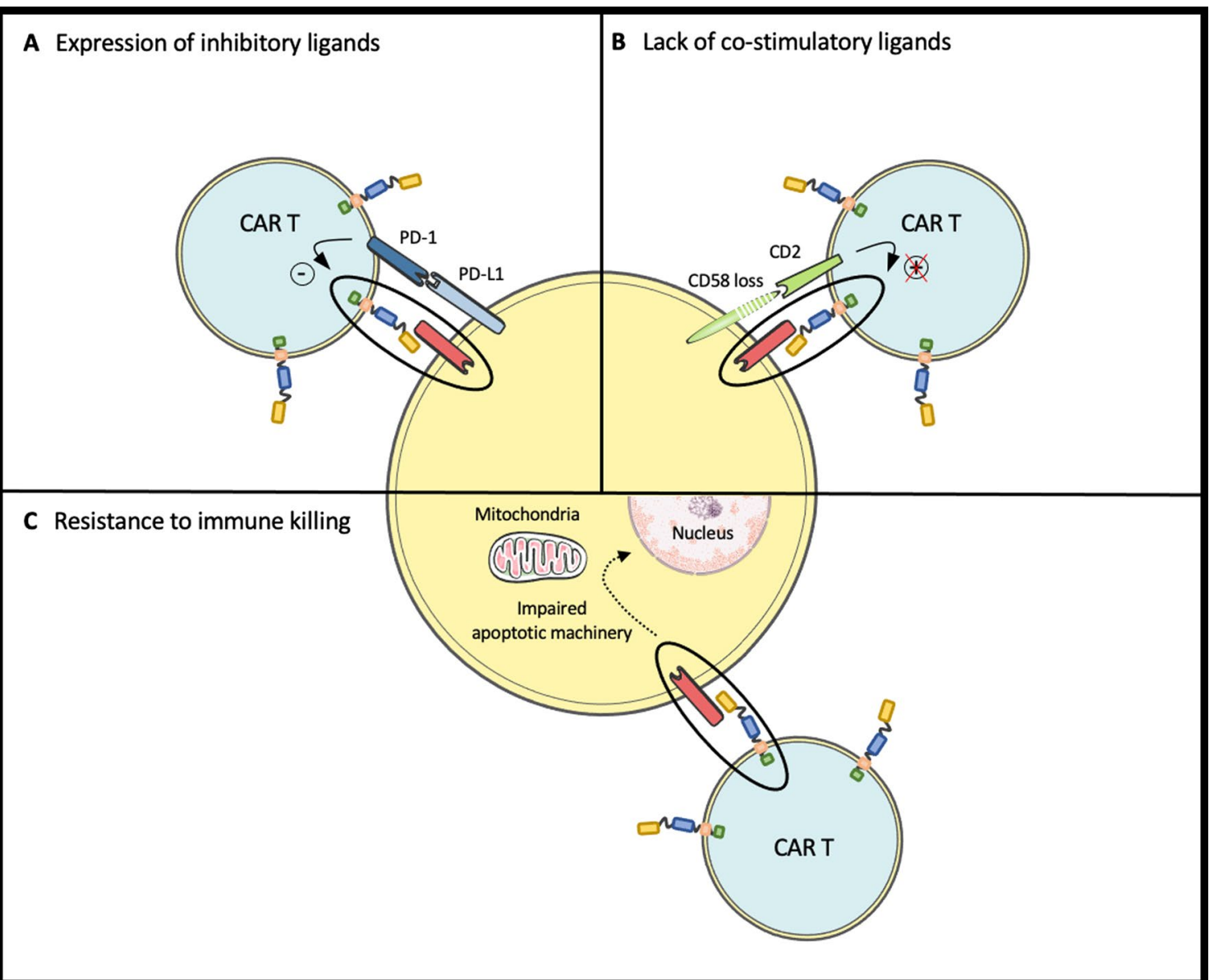

Fig. 2 Resistance mechanisms to CART cell therapy independent of target antigen loss. A Expression of inhibitory ligands (such as PD-L1) by tumor cells inhibit CART cell cytotoxicity despite target antigen recognition by CAR. B Lack of CD58 expression by tumor cells prevent CD2 to deliver a costimulatory to CART cell resulting in an impaired cytotoxicity despite target antigen recognition by CAR. C Impaired apoptotic machinery in tumor cells confer intrinsic tumor cell resistance to CART cell mediated immune killing despite target antigen recognition by CAR

\section{Epitope masking}

Ruella and colleagues reported the insertion of the CAR transgene into a single leukemic B cell during CAR T cell manufacturing. Expression of the CAR at the blast surface masked the CD19 epitope and thereby prevented the recognition of the tumor cell by CAR $T$ cells. This rare event has been observed in a single patient with B-ALL who relapsed 9 months after treatment with CD19 CAR $\mathrm{T}$ cell [31]. Most CAR T manufacturing protocols now include $\mathrm{T}$ cell selection to avoid this potential complication (Fig. 1D).

\section{Lineage switch and transdifferentiation}

Ag-loss may also be due to a lineage switch [32]. Unlike other mechanisms previously described, lineage switch is not only responsible for CD19 loss but results in a broader phenotypic switch resulting in the acquisition of acute myeloid leukemia (AML) markers. Two patients with MLL-rearranged B-ALL treated with CD19 CAR $T$ cells on the SCRI trial [33] and one patient from the Fred Hutchinson Cancer Center [10] were reported to experience a lineage switch with CD19-negative escape after CD19 CAR T cell therapy. As an exceptional event, MCL transdifferentiation into poorly differentiated sarcoma has been described after CD19 CAR T cells in one patient [34]. This transdifferentiation process was associated with a profound reprogramming of the epigenome responsible for tumor progression two months after CAR $\mathrm{T}$ cell infusion (Fig. 1E).

\section{Strategies to overcome Ag-low/negative relapses}

Ag-low relapses may be prevented by increasing the sensitivity of CAR T cells to their target whereas Ag-negative relapses may be overcome by targeting multiple Ags or by killing tumor cells in an Ag-independent manner. In 
Table 2 Therapies envisioned to circumvent resistance mechanisms due to cancer cells

\begin{tabular}{lll}
\hline Mechanism & Treatment & \\
\hline Loss of target-Ag & CAR-T construct & Higher affinity scFvs [35, 36] \\
& Tinge region [36] & CART cells with different specificities [37, 39-43] \\
& Target multiple antigens & Tandem or bicistronic CARs [44-49] \\
& Target tumor cells independently of the CART target & "Armored"CART cells [50-54] \\
& TRAlL-mediated death (low-dose radiation) [55] & Y-secretase inhibitors [57] \\
& Reducing antigen loss on target cell surface & Anti-CAR19 idiotype CART cells [58] \\
& Deplete transduced tumor cells in case of epitope masking & CART cells + anti-PD1 Ab [66-68] \\
Expression of inhibitory ligands (PD-L1) & Combination with CPI & Anti-PD1-secreting CART cells [69] \\
& & PD1-KO [70-72] \\
& PD-L1-resistant CART cells & PD1 dominant-negative receptor [73] \\
& & PD1 switch receptor [74] \\
& CAR with a CD2 signaling domain [77] \\
Lack of costimulatory ligand (CD58) & Provide CD2 costimulation independently of CD58 & DNA damaging agents [81] \\
& Enhance expression of death receptors & Histone deacetylase (HDAC) inhibitors [82, 85] \\
& & Proteasome inhibitors [83] \\
& & Cyclooxygenase-2 (COX2) inhibitors [84, 85] \\
& Smac mimetics (IAP inhibitors) [80] \\
\end{tabular}

both cases, strategies meant to re-induce or increase Agexpression may also be beneficial (Table 2).

\section{CAR T cell constructs with enhanced sensitivity to prevent low-Ag relapses}

CAR $\mathrm{T}$ cells with higher affinity single-chain variable fragments ( $\mathrm{scFvs}$ ) have been developed to recognize Ags expressed at a low density. In vitro, CAR T cells harboring a high-affinity $\mathrm{scFv}$ were able to recognize their target when expressed at very low levels, including when the target was undetectable by flow cytometry [35]. Furthermore, adding immunoreceptor tyrosine-based activation motifs (ITAM) enhanced the strength of intracellular signaling, both in CD28 and 4-1BB CAR T cells, and thus enabled recognition of tumor cells with a low Ag-density [36]. Finally, the hinge region may impact the ability of CAR T cells to bind its epitope. As an example, replacement of the CD8 hinge-transmembrane region of a 4-1BB $\zeta$ CAR with a CD28-hinge-transmembrane region lowered the threshold for CAR reactivity and enhanced the killing of CD19-low leukemic cells [36]. These approaches may be particularly beneficial in the context of CD22 and BCMA CAR T cells because patients often relapse with CD22dim [37, 38] or BCMAdim [22] upon these immunotherapies whereas relapses upon CD19 CAR T cells mostly exhibit complete CD19 loss.

\section{CAR T cells targeting multiple Ags to prevent Ag-negative relapses}

As discussed above, recent data suggest that prior to any treatment, a significant portion of patients harbor preexisting Ag-negative tumor cells. This initial tumor heterogeneity may lead to relapse upon CAR $\mathrm{T}$ cell therapy. To overcome Ag-negative relapses, CAR T cells directed against multiple Ags have been developed [39-41]. This may be achieved by infusing a cocktail of CAR T cells with unique but different specificities or by infusing CAR T cells with multiple specificities.

CAR T cells with different specificities can be administered simultaneously or sequentially to prevent Agnegative relapses. In a phase I trial, $21 \mathrm{~B}$-ALL patients were treated with CD22 CAR T cells including 17 who had been previously treated with a CD19-directed immunotherapies [37]. CD22 is expressed in most cases of B-ALL and is usually retained following CD19 loss. In this study, all 5 patients with a CD19dim or CD19-negative B-ALL achieved a complete remission after receiving $>1 \times 10^{6}$ CD22 CAR $\mathrm{T}$ cells. The median duration of response was 6 months. Interestingly, relapses were associated with decreased CD22 expression by tumor cells. Similarly, Baird et al. reported 3 patients with CD19 CAR resistant DLBCL who were treated with CD22 CAR $\mathrm{T}$ cells and achieved a complete remission [42]. From 
these observations, Pan and colleagues hypothesized that a sequential administration upfront of 2 CAR $T$ cell products targeting different Ags may improve longterm outcomes. They conducted a phase 1 trial evaluating a sequential administration of CD19 and CD22 CAR $\mathrm{T}$ cells in pediatric patients with R/R B-ALL [43]. In this trial, 17 (85\%) of the 20 patients treated with sequential CAR T cells remained in CR at the end of the study and 3 relapsed at 6.6, 6.9 and 11.4 months, resulting in a 1-year PFS and OS of $79.5 \%$ and $92.3 \%$, respectively. Among relapsed patients, one exhibited CD22 downregulation on leukemic cells and CD19 Ag-loss was observed in 2 patients.

CAR T cells with multiple specificities have also been developed which can be i) tandem (or bivalent) CARs with two distinct Ag-binding sites on a single extracellular domain, or ii) bicistronic CARs which are engineered using a single vector encoding two distinct CARs to allow dual targeting through separate extracellular motifs. CD19/CD22 tandem CAR constructs were administered to 12 patients with R/R B-ALL: 11 achieved a CR and 1 experienced primary progressive disease with CD19 retention [44]. In a recent study focusing on adult $R / R$ B-ALL, 6 out of 6 infused patients achieved an MRDnegative CR after infusion with tandem CD19/CD22 CAR T cells. Of note, one patient experienced a relapse at 5 months post-infusion with CD19-negative and CD22low blasts [45]. Such strategies have also been applied to $\mathrm{R} / \mathrm{R}$ aggressive $\mathrm{B}$ lymphomas. In a phase I dose-escalation study, 11 patients (5 DLBCL, $4 \mathrm{MCL}, 2 \mathrm{CLL}$ ) were treated with CD19/CD20 tandem CAR T cells [46]. The ORR was $82 \%$ at day 28 (6/11 CR and 3/11 PR). All progressing patients retained either CD19 or CD20 positivity suggesting alternative resistance mechanisms to Ag-loss. Toxicity was acceptable [47]. The AUTO3 trial evaluated a bicistronic CAR T cell targeting both CD19 and CD22 in pediatric R/R B-ALL. All 7 evaluable patients achieved a CR/CRi following CAR $T$ cells infusion with a negative minimal residual disease (MRD) [48]. Three relapses were reported including one with CD19 negative/CD22 low expression. Thus, Ag escape may be observed as a resistance mechanism even upon multi-targeted strategies. This bicistronic construct has also been evaluated in the phase I Alexander study for the treatment of $R / R$ DLBCL, combined with the anti-PD1 antibody, pembrolizumab [49]. In this study, among the 11 patients treated at a dose $>50 \times 10^{6}$, the ORR and CRR were $64 \%$ and $55 \%$, respectively. Toxicities were acceptable and in line with CAR T cells targeting a single Ag.

\section{CAR T cells to kill cancer cells in an Ag-independent manner}

"Armored" CAR T cells have been developed to secrete immune stimulatory cytokines (e.g., IL12 or IL18) or express costimulatory ligands (e.g., CD40L, Flt3L, or 4-1BBL) in order to enhance CAR T cell efficacy ("auto/ transactivation") and/or generate/recruit natural antitumor T cells ("epitope spreading").

Armored CAR T cells secreting IL12 and IL18 demonstrated enhanced activity in preclinical models. IL12 secretion by CAR $\mathrm{T}$ cells resulted in increased antitumor efficacy [50]. IL12 can increase the antitumor function of CAR T cells in an autocrine manner. It can also shape the tumor microenvironment, rendering CAR $\mathrm{T}$ cells resistant to regulatory $\mathrm{T}$ cells and myeloid-derived suppressor cells immunosuppression. Avanzi and colleagues designed armored IL18-secreting CAR T cells, which increased IFN- $\gamma$ secretion and tuned the tumor microenvironment toward an IFN- $\gamma$ signature [51]. These IL18secreting CAR $\mathrm{T}$ cells were able to modulate the tumor microenvironment and enhance the endogenous antitumor immune response.

Kuhn and colleagues developed CAR T cells expressing CD40L constitutively. This construct enables CD40L expressed on the CAR $\mathrm{T}$ cells to engage with CD40positive tumor cells (resulting in direct cytotoxicity) and with antigen-presenting cells (APC). CD40L-CD40 interaction triggered activation of tumor-adjacent APC and increased expression of costimulatory molecules such as CD40, CD86 and major histocompatibility complex (MHC) class II. Thus, constitutive expression of CD40L by CAR $T$ cells can generate endogenous antitumor $\mathrm{T}$ cells which can recognize and kill tumor cells through their TCR, thereby preventing the risk of immune escape via the loss of a single Ag [52]. Similarly, Lai et al. engineered $T$ cells to secrete the dendritic cell (DC) growth factor Fms-like tyrosine kinase 3 ligand (Flt3L) [53]. This construct induced the activation of endogenous $\mathrm{T}$ cells, enabling a broader repertoire of tumor Ags to be targeted via the expansion of intratumoral APCs, significantly improving tumor responses.

Costimulatory ligands such as CD80 and 4-1BBL may also be expressed on CAR T cells to stimulate bystander endogenous and CAR T cells (transactivation). CD80 and 4-1BBL bind to two costimulatory receptors expressed on T cells, CD28 and 4-1BB, respectively. Primary human $\mathrm{T}$ cells overexpressing CD80 and 4-1BBL were shown to eradicate tumor cells very efficiently, even in the absence of costimulatory ligands [54]. CD80/4-1BBL expressing CTL were able to induce trans-costimulation of bystander $\mathrm{T}$ cells. These strategies could be applied to CAR T cells. By stimulating endogenous $\mathrm{T}$ cells locally, they may promote/enhance tumor cell killing beyond their target-Ag.

Alternative death signaling pathways may also be used to enable CAR T cells to kill cancer cells independently of their target. For instance, low-dose radiation 
induces expression of death receptors such as TRAILreceptors on the tumor cells' surface. Thus, tumor cells are sensitized to TRAIL-mediated apoptosis by CAR T cells. CAR T cells activated by Ag-positive tumor cells can then induce bystander killing of Ag-negative tumor cells through the death receptors pathway. This strategy increased both on-target and bystander CAR T cell cytotoxicity, enabling tumor control even in the case of Agloss variants [55].

\section{Reducing Ag-loss on target cell surface}

Although CAR $\mathrm{T}$ cells may exert their action independently of their target-Ag, their efficacy is thought to be tightly associated with the density of target-Ags on the cell membrane. Thus, combining CAR $\mathrm{T}$ cells with treatments that increase target expression on the tumor cells could be of interest. Efficacy of BCMA CAR T cells for the treatment of multiple myeloma is thought to be limited by BCMA cleavage from the tumor cell surface by a $\gamma$-secretase, which decreases ligand density on myeloma cells [56]. In murine models, inhibition of the $\gamma$-secretase activity reduced Ag-loss on the target cell and improved antitumor efficacy of BCMA CAR T cells [57]. Based on these data, clinical trials evaluating the combination of $\gamma$-secretase inhibition with concurrent BCMA CAR T cell treatment are ongoing (NCT03502577).

\section{Depleting tumor cells in case of epitope masking}

Unintentional transduction of B-ALL blasts during CD19 CAR T cells manufacturing can lead to CD19 CAR T cells treatment resistance through epitope masking. AntiCD19 CAR idiotype CAR has been developed to specifically recognize and deplete transduced B-ALL blasts [58].

\section{Expression of inhibitory ligands (PD-L1 expression)}

The programmed death-1 (PD-1)/programmed death-1 ligand-1 (PD-L1) axis is a well-known immune checkpoint inhibitor pathway. The inhibitory ligands PD-L1 and PD-L2 may be expressed by tumor cells or their microenvironment. These ligands prevent $T$ cell activation upon binding to their receptor (i.e., PD-1), allowing immune escape (Fig. 2A).

\section{Incidence}

PD-L1/L2 have been reported to be expressed in B cell malignancies. In R/R DLBCL, $16 \%$ of all evaluable samples exhibited low-level copy gain and $3 \%$ had amplification of 9p24.1, the locus encoding for PD-L1 and PD-L2 $[59,60]$. By immunohistochemistry, 4 out of 46 cases (9\%) showed membrane expression of PD-L1 on biopsy specimens. Primary mediastinal large B cell lymphoma (PMBCL) has been reported to be frequently associated with genetic aberrations at $9 \mathrm{p} 24$, resulting in tumor expression of PD-L1 and PD-L2 [61]. Finally, PD-L1 has also been reported to be expressed in a subset of B-ALL $[62,63]$.

\section{Mechanisms}

PD-1 is expressed on activated CAR T cells [64]. PD-L1 expression by the tumor cells or the tumor microenvironment may inhibit CAR T cell cytotoxicity and induce immune resistance. Neelapu et al. demonstrated that 13 (62\%) out of 21 DLBCL patients who had progressed after Axi-Cel therapy in the ZUMA-1 trial expressed PD-L1[65]. Hence, unleashing the inhibition of immune checkpoints such as PD-1 may enhance the efficacy of CAR T cell therapy.

\section{Strategies to overcome PD-L1-mediated resistance}

Several strategies may be envisioned to overcome PDL1-mediated immune suppression, including combination of CAR $\mathrm{T}$ cells with anti-PD1/PD-L1 antibodies, anti-PD1-secreting CAR T cells, or PD-L1-resistant CAR (Table 2).

\section{Combination of anti-PD1/PD-L1 antibodies with CAR T cells}

The combination of an immune checkpoint blocker with CAR $\mathrm{T}$ cells has been evaluated in 2 different setting: $\mathrm{i}$ ) at the time of relapse after CAR T cell therapy and ii) at the time of CAR T cell infusion (i.e., upfront). Thus far, only small cohorts of patients have been treated with this combination. In a single-center study, 12 DLBCL patients in relapse or progression after CD19 CAR T cells received an anti-PD1 antibody (Ab), pembrolizumab [66]. Among 11 evaluable patients, 1 achieved a complete response, 2 a partial response, 1 a stable disease, and 7 progressed. Interestingly, 9 of the 12 patients showed a re-expansion of the CAR T cells as measured by transgene copy number. However, no correlation could be made between the peak of CAR T cells and the clinical response in this small cohort. Anti-PD1 antibodies have also been combined with CAR $\mathrm{T}$ cells at the time of adoptive transfer. In a small study, 6 DLBCL patients received the anti-PDL1 Ab durvalumab 21-28 days after CAR T cells infusion, and 9 patients were treated with durvalumab the day before infusion [67]. The overall response rate was $50 \%$. With a median follow-up of 10.6 months, only one patient among the 5 patients who achieved a CR has relapsed. In this study, 5/13 patients developed a cytokine release syndrome (CRS) including one grade 4 , and one patient experienced a neurotoxicity. In the ZUMA-6 study, CD19 CAR T cells were given in combination with the anti-PD-L1 Ab atezolizumab for the treatment of R/R DLBCL [68]. In this study, atezolizumab was administered starting either on day 21,14 or 1 post CAR $T$ cell infusion. The most common grade 3-4 AEs were anemia 
(9/12), encephalopathy (5/12), and neutropenia (5/12). Grade 3-4 CRS and neurotoxicity occurred in 3/12 and $6 / 12$ patients, respectively. After a median follow-up of 4.4 months, 9 out of 10 patients experienced an objective response, including $6 \mathrm{CR}$ and $3 \mathrm{PR}$. The ALEXANDER trial evaluated the combination of a bispecific CD19/ CD22 CAR T cell (Auto3) and pembrolizumab [49]. Among 11 patients treated at a dose $>50 \times 10^{\wedge} 6$, the ORR and CRR were $64 \%$ and $55 \%$, respectively. At this dose level, there were no case of severe CRS nor neurotoxicity of any grade. These observations indicate that combination strategies of immune checkpoint blockers with CAR $\mathrm{T}$ cells may be beneficial for a subset of patients and suggest that other resistance pathways might play a crucial role.

\section{Anti-PD1-secreting CAR T cells}

Rafiq and colleagues developed "armored" CAR T cells capable of secreting PD1 blocking scFv which can act both in a paracrine and autocrine manner [69]. In murine models, the efficacy of these CAR T cells against PDL1-expressing tumors was at least as good as the combination of CAR T cells with an anti-PD1 Ab. Such CAR $\mathrm{T}$ cells allow local delivery of anti-PD1 scFv which could limit the toxicities of a systemic exposure to checkpoint inhibitors.

\section{PD-L1-resistant CART cells}

CAR T cells have been engineered to be resistant to PD-1 signaling. This can be achieved by knocking-out the PD1 gene using the CRISR-Cas9 technology [70-72] or by transducing a PD1 dominant-negative receptor [73]. CAR $\mathrm{T}$ cells can also be transduced with a PD1/CD28 chimeric switch receptor. This receptor contains the extracellular domain of PD1 fused with the transmembrane and cytoplasmic domain of the costimulatory molecule, CD28. Ligation of PD-L1 to its receptor (PD1) transmits an activating signal (via the CD28 cytoplasmic domain) instead of the inhibitory signal normally transduced by the PD1 cytoplasmic domain [74]. These CAR T- cells have been shown to resist PD1 mediated inhibition in preclinical models. However, no clinical data are yet available with these constructs.

\section{Lack of costimulatory ligand (CD58 loss)}

CD58 is the ligand for the CD2 molecule expressed on human $\mathrm{T}$ cells. CD2 provides a costimulatory signal for $\mathrm{T}$ cell proliferation, cytokine production and activation via TCR signaling $[75,76]$. Similar to natural cytotoxic $\mathrm{T}$ cells, CD2 ligation is crucial for CAR activation and cytoskeletal rearrangement required for tumor cell killing (Fig. 2B).

\section{Incidence}

CD58 alteration (mutation or loss of expression) is seen in approximately a quarter of DLBCL. Furthermore, CD58 alteration is associated with a worse outcome after CD19 CAR T cell treatment [77].

\section{Mechanisms}

CD58 alteration may be due to mutations or lack of expression as measured by IHC [77]. CD58 alteration decreases CAR $\mathrm{T}$ cell activation and cytotoxicity in preclinical models.

\section{Strategies to overcome lack of costimulatory ligands}

Lack of CD58-CD2 signaling may be overcome by adding a second CAR construct containing a CD2-signaling domain integrated in the cytoplasmic tail of the CAR (Table 2). CAR binding to its target-Ag will induce CD2 signaling independently of CD58 expression by the tumor cells. Such construct demonstrated increased activity in preclinical models but have not yet been tested in patients [77].

\section{Resistance to immune killing}

Tumor cells may be resistant to immune cell killing by CAR $\mathrm{T}$ cells by a mechanism called "intrinsic resistance" (Fig. 2C). This specific mechanism of resistance has been recently reviewed in details elsewhere [78].

\section{Incidence}

The intrinsic resistance of tumor cells to CAR T cell killing has been recently reported. However, its prevalence in hematologic malignancies remains unknown.

\section{Mechanisms}

Recently, accumulating data revealed that impaired apoptosis machinery in tumor cells could render tumor cells resistant to immune killing by CAR T cells. Two unbiased genome-wide loss-of-function screens in B-ALL and B cell lymphoma cell lines revealed the crucial role of the death receptor pathway of apoptosis to mediate CAR T cell cytotoxicity $[79,80]$. These studies demonstrated that disruption of genes associated with pro-apoptotic death receptor signaling pathway such as FADD, BID, CASP8, and TNFRSF10B conferred resistance to CAR T cell killing. Conversely, knock-out of anti-apoptotic genes such as CFLAR, TRAF2, and BIRC2 led to an increased susceptibility to CAR $\mathrm{T}$ cell killing. Using samples from two multicenter trials of relapsed/refractory pediatric and adult B-ALL, Singh and colleagues found a significantly higher death receptor signaling signature in samples from patients who had achieved complete remissions compared to patients who did not respond despite retaining CD19 expression [79]. 


\section{Strategies to overcome intrinsic resistance to CAR T cell killing}

Based on these observations, strategies have been considered to sensitize tumor cells to death receptor mediated apoptosis (Table 2). Lowering the threshold necessary to induce this type of cellular death may indeed overcome intrinsic resistance of tumor cells to CAR T cell therapy. Several cancer therapeutic agents may modulate death receptor expression by tumor cells including DNA damaging agents [81], histone deacetylase (HDAC) inhibitors [82], proteasome inhibitors [83] and cyclooxygenase-2 (COX2) inhibitors [84]. In preclinical models, HDAC inhibitors (SAHA and LBH589) and COX2 inhibitors (celecoxib) were able to partially reverse CD19 CAR T cell resistance of non-Hodgkin lymphoma cell lines due to altered death receptor pathway machinery [85]. To identify novel candidate molecules, Dufva and colleagues carried out a high-throughput drug screen using a coculture assay with CD19-directed CAR T cells in presence of the CD19-positive B-ALL cell line, NALM6. Strikingly, the three drugs that most significantly enhanced CAR T cell cytotoxicity all belonged to the same pharmacological class, namely second mitochondrial-derived activator of caspases (Smac) mimetics or inhibitor of apoptosis proteins (IAP) inhibitors (Birinapant, AT-406, and LCL161) [80]. Another pharmacologic class which may be of interest are the B cell lymphoma 2 (Bcl-2) inhibitors. $\mathrm{Bcl}-2$ prevents apoptosis. Its overexpression can promote tumor cells survival and may lead to treatment resistance. Thus, the combination of Bcl-2 inhibitors with CAR T cells may be an attractive strategy. In a coculture model containing B-ALL and CD19 CAR T cells, Bcl-2 inhibition decreased the apoptosis threshold in leukemic cells leading to an enhanced CAR T cell cytotoxicity [86]. Moreover, in vitro pre-sensitization of B-ALL cells with the Bcl-2 inhibitor venetoclax resulted in an enhanced CAR $\mathrm{T}$ cell mediated cytotoxicity by upregulating the CD19 expression and pro-apoptotic proteins [87]. However, these strategies are yet to be tested in clinic.

\section{Conclusion}

Understanding how tumor cells resist CAR T cell therapy is a crucial step toward the development of strategies to improve CAR T cell efficacy. Fundamental and clinical studies on evasions to monoclonal antibody treatment were instructive as some resistance mechanisms are shared with CAR $\mathrm{T}$ cells given that both treatments are responsible for a selection pressure on a specific tumor marker. Indeed, loss of target antigen was previously reported as a resistance pathway to monoclonal antibodies or BiTE as rituximab for CD20, blinatumomab for CD19 and inotuzumab ozogamicin for CD22 [30, 88-90].
Thus, in the CAR T cell field, loss of target-Ag has been the best-characterized mechanism of resistance, and new approaches are being developed to overcome or prevent this issue, including CAR T cells directed against multiple Ags. Recent studies unveiled other resistance mechanisms developed by cancer cells to evade eradication by CAR T- cells, including expression of inhibitory ligands, lack of costimulatory ligands, and intrinsic resistance to CAR-T killing. Each of these resistance mechanisms may be circumvented by strategies directed toward the CAR T cells or the tumors cells. However, most of these strategies remain to be evaluated in patients. From a broader perspective, one shall remember that three compartments may contribute to CAR $\mathrm{T}$ cell resistance: the tumor cells (reviewed here), the tumor microenvironment, and the CAR T cells. The role of tumor microenvironment may be particularly important in solid tumors due to the extracellular matrix and cytokine milieu present in non-hematopoietic tissues [91-93]. Thus, novel CAR $T$ cell designs should address the resistance mechanisms of all three compartments. Finally, as this field is moving forward, it will become increasingly important to characterize the resistance mechanisms of each individual tumor in order to personalize CAR T cell therapy with the optimal product or combination.

\section{Abbreviations \\ CAR: Chimeric antigen receptor; FDA: Food and drug administration; B-ALL: B cell acute lymphoblastic leukemia; DLBCL: Diffuse large B cell lymphoma; $M C L$ : Mantle cell lymphoma; FL: Follicular lymphoma; MM: Multiple myeloma; Ag: Antigen; BCMA: B cell maturation antigen; R/R: Relapsed or refractory; CHOP. Children's Hospital of Philadelphia; BiTE: Bispecific T cell engager; AML: Acute myeloid leukemia; scFvs: Single-chain variable fragments; ITAM: Immuno- receptor tyrosine-based activation motifs; MRD: Minimal residual disease; APC: Antigen-presenting cells; MHC: Major histocompatibility complex; DC: Dendritic cell; Flt3L: Fms-like tyrosine kinase 3 ligand; PD-1: Programmed death-1; PD-L1: Programmed death-1 ligand-1; PMBCL: Primary mediastinal large B cell lymphoma; Ab: Antibody; CRS: Cytokine release syndrome; HDAC: Histone deacetylase; COX2: Cyclooxygenase-2; Smac: Second mitochondrial- derived activator of caspases; IAP: Inhibitor of apoptosis proteins; BCI-2: B cell lymphoma 2.}

\section{Acknowledgements}

Not applicable.

\section{Authors' contributions}

$J L$ wrote the manuscript; $J L, M R$ and $R H$ approved the final version of the manuscript and are accountable for all aspects of the work. All authors read and approved the final manuscript.

\section{Funding}

M.R. was supported by: Lymphoma research foundation (LRF) CDA (M.R.); Gilead Research Scholar Award (M.R.); Gabrielle's Angel Foundation (M.R.); Laffey-McHugh Foundation; Emerson Collective Award (M.R.); LaffeyMcHugh Foundation (M.R.); Parker Institute for Cancer Immunotherapy (M.R.); Berman and Maguire Funds for Lymphoma Research at Penn (M.R.); NCI 1K99CA212302 and R00CA212302 (M.R.).

Availability of data and materials Not applicable. 


\section{Declarations}

Ethics approval and consent to participate

Not applicable.

\section{Consent for publication}

Not applicable.

\section{Competing interests}

$J$ has nothing to disclose. M.R. holds patents related to CART cells that are managed to the University of Pennsylvania and licensed to Novartis, Tmunity and viTToria biotherapeutics. M.R. has served as a consultant for nanoString, BMS, GSK, Bayer, and AbClon. M.R. receives research funding from AbClon, nanoString and Beckam Coulter. M.R. is the scientific founder of viTToria biotherapeutics. $\mathrm{RH}$ received honoraria from Bristol-Myers Squibb, MSD, Gilead, Kite, Roche, Novartis, Janssen, and Celgene.

\section{Author details}

${ }^{1}$ Department of Hematology, AP-HP, Université de Paris, Paris, France. ${ }^{2}$ Center for Cellular Immunotherapies and Division of Hematology-Oncology, University of Pennsylvania, Philadelphia, PA, USA. ${ }^{3}$ Department of Hematology, CHU de Rennes, Université de Rennes, INSERM U1236, 2 rue Henri Le Guilloux, 35033 Rennes Cedex 9, France.

Received: 24 August 2021 Accepted: 4 November 2021

Published online: 22 November 2021

\section{References}

1. Chong EA, Ruella M, Schuster SJ, Lymphoma Program Investigators at the University of Pennsylvania. Five-year outcomes for refractory B-cell lymphomas with CAR T-cell therapy. N Engl J Med. 2021;384:673-4.

2. Ghilardi G, Braendstrup P, Chong EA, Schuster SJ, Svoboda J, Ruella M. CAR-T TREK through the lymphoma universe, to boldly go where no other therapy has gone before. Br J Haematol. 2021;193:449-65.

3. Majzner RG, Mackall CL. Tumor antigen escape from CAR T-cell therapy. Cancer Discov. 2018;8:1219-26.

4. Larson RC, Maus MV. Recent advances and discoveries in the mechanisms and functions of CAR T cells. Nat Rev Cancer. 2021;21:145-61.

5. Ruella M, Maus MV. Catch me if you can: leukemia escape after CD19-directed T cell Immunotherapies. Comput Struct Biotechnol J. 2016:14:357-62.

6. Park JH, Rivière I, Gonen M, Wang X, Sénéchal B, Curran KJ, et al. Longterm follow-up of CD19 CAR therapy in acute lymphoblastic leukemia. N Engl J Med. 2018;378:449-59.

7. Maude SL, Laetsch TW, Buechner J, Rives S, Boyer M, Bittencourt H, et al. Tisagenlecleucel in children and young adults with B-cell lymphoblastic leukemia. N Engl J Med. 2018;378:439-48.

8. Maude SL, Frey N, Shaw PA, Aplenc R, Barrett DM, Bunin NJ, et al. Chimeric antigen receptor $T$ cells for sustained remissions in leukemia. N Engl J Med. 2014;371:1507-17.

9. Gardner RA, Finney O, Annesley C, Brakke H, Summers C, Leger $\mathrm{K}$, et al. Intent-to-treat leukemia remission by CD19 CART cells of defined formulation and dose in children and young adults. Blood. 2017;129:3322-31.

10. Turtle CJ, Hanafi L-A, Berger C, Gooley TA, Cherian S, Hudecek M, et al. CD19 CAR-T cells of defined CD4+:CD8+ composition in adult B cell ALL patients. J Clin Invest. 2016;126:2123-38.

11. Dourthe M-E, Rabian F, Yakouben K, Chevillon F, Cabannes-Hamy A, Méchinaud F, et al. Determinants of CD19-positive vs CD19-negative relapse after tisagenlecleucel for B-cell acute lymphoblastic leukemia. Leukemia. 2021

12. Neelapu SS, Locke FL, Bartlett NL, Lekakis LJ, Miklos DB, Jacobson CA, et al. Axicabtagene ciloleucel CAR T-cell therapy in refractory large B-cell lymphoma. N Engl J Med. 2017;377:2531-44.

13. Schuster SJ, Svoboda J, Chong EA, Nasta SD, Mato AR, Anak Ö, et al. Chimeric antigen receptor $T$ cells in refractory B-cell lymphomas. N Engl J Med. 2017;377:2545-54.
14. Neelapu SS, Rossi JM, Jacobson CA, Locke FL, Miklos DB, Reagan PM, et al. CD19-loss with preservation of other B cell lineage features in patients with large B cell lymphoma who relapsed post-axi-cel. Blood. 2019;134:203-203.

15. Spiegel JY, Dahiya S, Jain MD, Nastoupil LJ, Ghobadi A, Lin Y, et al. Outcomes in large B-cell lymphoma progressing after axicabtagene ciloleucel (Axi-cel): Results from the U.S. Lymphoma CAR-T Consortium. J Clin Oncol Wolters Kluwer. 2019;37:7517-7517.

16. Yu H, Sotillo E, Harrington CT, Wertheim G, Paessler M, Maude S, et al. Repeated loss of target surface antigen after immunotherapy in primary mediastinal large B cell lymphoma. undefined [Internet]. 2017 [cited 2021 May 22]; Available from: /paper/Repeated-loss-of-targetsurface-antigen-after-in-B-Yu-Sotillo/2c646f524dd4ceacfe5aff505b793e $630 \mathrm{bf} 23 \mathrm{bfd}$

17. Shalabi H, Kraft IL, Wang H-W, Yuan CM, Yates B, Delbrook C, et al. Sequential loss of tumor surface antigens following chimeric antigen receptor T-cell therapies in diffuse large B-cell lymphoma. Haematologica. 2018;103:e215-8.

18. Plaks V, Rossi JM, Chou J, Wang L, Poddar S, Han G, et al. CD19 target evasion as a mechanism of relapse in large B-cell lymphoma treated with axicabtagene ciloleucel. Blood. 2021;138:1081.

19. Wang M, Munoz J, Goy A, Locke FL, Jacobson CA, Hill BT, et al. KTE-X19 CAR T-cell therapy in relapsed or refractory mantle-cell lymphoma. $\mathrm{N}$ Engl J Med. 2020:382:1331-42.

20. Plaks V, Chou J, Goyal L, Zhang W, Salunkhe S, Sehgal AR, et al. Abstract CT036: Axicabtagene ciloleucel (axi-cel) product attributes and immune biomarkers associated with clinical outcomes in patients (pts) with relapsed/refractory (R/R) indolent non-Hodgkin lymphoma (iNHL) in ZUMA-5. Cancer Res. 2021;81:CT036.

21. Ali SA, Shi V, Maric I, Wang M, Stroncek DF, Rose JJ, et al. T cells expressing an anti-B-cell maturation antigen chimeric antigen receptor cause remissions of multiple myeloma. Blood. 2016;128:1688-700.

22. Cohen AD, Garfall AL, Stadtmauer EA, Melenhorst JJ, Lacey SF, Lancaster $E$, et al. B cell maturation antigen-specific CART cells are clinically active in multiple myeloma. J Clin Invest. 2019:129:2210-21.

23. Rosenthal J, Naqvi AS, Luo M, Wertheim G, Paessler M, Thomas-Tikhonenko A, et al. Heterogeneity of surface CD19 and CD22 expression in B lymphoblastic leukemia. Am J Hematol. 2018;93:E352-5.

24. Cheng J, Zhao L, Zhang Y, Qin Y, Guan Y, Zhang T, et al. Understanding the mechanisms of resistance to CAR T-cell therapy in malignancies. Front Oncol. 2019;9:1237.

25. Pillai V, Muralidharan K, Meng W, Bagashev A, Oldridge DA, Rosenthal J, et al. CAR T-cell therapy is effective for CD19-dim B-lymphoblastic leukemia but is impacted by prior blinatumomab therapy. Blood Adv. 2019;3:3539-49.

26. Orlando EJ, Han X, Tribouley C, Wood PA, Leary RJ, Riester M, et al. Genetic mechanisms of target antigen loss in CAR19 therapy of acute lymphoblastic leukemia. Nat Med. 2018;24:1504-6.

27. Zhang Z, Chen X, Tian Y, Li F, Zhao X, Liu J, et al. Point mutation in CD19 facilitates immune escape of B cell lymphoma from CAR-T cell therapy. J Immunother Cancer BMJ Specialist J. 2020;8:e001150.

28. Sotillo E, Barrett DM, Black KL, Bagashev A, Oldridge D, Wu G, et al. Convergence of acquired mutations and alternative splicing of CD19 enables resistance to CART-19 immunotherapy. Cancer Discov. 2015:5:1282-95.

29. Fischer J, Paret C, El Malki K, Alt F, Wingerter A, Neu MA, et al. CD19 isoforms enabling resistance to CART-19 immunotherapy are expressed in B-ALL patients at initial diagnosis. J Immunother Hagerstown Md. 1997;2017(40):187-95.

30. Braig F, Brandt A, Goebeler M, Tony H-P, Kurze A-K, Nollau P, et al. Resistance to anti-CD19/CD3 BiTE in acute lymphoblastic leukemia may be mediated by disrupted CD19 membrane trafficking. Blood. 2017;129:100-4

31. Ruella M, Xu J, Barrett DM, Fraietta JA, Reich TJ, Ambrose DE, et al. Induction of resistance to chimeric antigen receptor $T$ cell therapy by transduction of a single leukemic B cell. Nat Med. 2018;24:1499-503.

32. Jacoby E, Nguyen SM, Fountaine TJ, Welp K, Gryder B, Qin H, et al. CD19 CAR immune pressure induces B-precursor acute lymphoblastic leukaemia lineage switch exposing inherent leukaemic plasticity. Nat Commun. 2016;7:12320. 
33. Gardner R, Wu D, Cherian S, Fang M, Hanafi L-A, Finney O, et al. Acquisition of a CD19-negative myeloid phenotype allows immune escape of MLL-rearranged B-ALL from CD19 CAR-T-cell therapy. Blood. 2016;127:2406-10.

34. Zhang Q, Orlando EJ, Wang HY, Bogusz AM, Liu X, Lacey SF, et al. Transdifferentiation of lymphoma into sarcoma associated with profound reprogramming of the epigenome. Blood. 2020;136:1980-3.

35. Liu X, Jiang S, Fang C, Yang S, Olalere D, Pequignot EC, et al. Affinitytuned ErbB2 or EGFR chimeric antigen receptor T cells exhibit an increased therapeutic index against tumors in mice. Cancer Res. 2015;75:3596-607.

36. Majzner RG, Rietberg SP, Sotillo E, Dong R, Vachharajani VT, Labanieh $L$, et al. Tuning the antigen density requirement for CAR T-cell activity. Cancer Discov. 2020;10:702-23.

37. Fry TJ, Shah NN, Orentas RJ, Stetler-Stevenson M, Yuan CM, Ramakrishna $\mathrm{S}$, et al. CD22-targeted CART cells induce remission in B-ALL that is naive or resistant to CD19-targeted CAR immunotherapy. Nat Med. 2018;24:20-8.

38. Singh N, Frey NV, Engels B, Barrett DM, Shestova O, Ravikumar P, et al. Antigen-independent activation enhances the efficacy of 4-1BBcostimulated CD22 CAR T cells. Nat Med. 2021;27:842-50.

39. Ruella M, Barrett DM, Kenderian SS, Shestova O, Hofmann TJ, Perazzelli J, et al. Dual CD19 and CD123 targeting prevents antigen-loss relapses after CD19-directed immunotherapies. J Clin Invest. 2016;126:3814-26.

40. Cronk RJ, Zurko J, Shah NN. Bispecific Chimeric Antigen Receptor T Cell Therapy for B Cell Malignancies and Multiple Myeloma. Cancers. Multidisciplinary Digital Publishing Institute; 2020;12:2523.

41. Shah NN, Maatman T, Hari P, Johnson B. Multi targeted CAR-T cell therapies for B-cell malignancies. Front Oncol. 2019;9:146.

42. Baird JH, Frank MJ, Craig J, Patel S, Spiegel JY, Sahaf B, et al. CD22directed CAR T-cell therapy induces complete remissions in CD19directed CAR-refractory large B-cell lymphoma. Blood. 2021;137:2321-5.

43. Pan J, Zuo S, Deng B, Xu X, Li C, Zheng Q, et al. Sequential CD19-22 CAR $T$ therapy induces sustained remission in children with $r / r$ B-ALL. Blood. 2020;135:387-91.

44. Schultz LM, Muffly LS, Spiegel JY, Ramakrishna S, Hossain N, Baggott C, et al. Phase I trial using CD19/CD22 bispecific CAR T cells in pediatric and adult acute lymphoblastic leukemia (ALL). Blood. 2019;134:744-744

45. Dai H, Wu Z, Jia H, Tong C, Guo Y, Ti D, et al. Bispecific CAR-T cells targeting both CD19 and CD22 for therapy of adults with relapsed or refractory B cell acute lymphoblastic leukemia. J Hematol Oncol. 2020;13:1-10

46. Shah NN, Johnson BD, Schneider D, Zhu F, Szabo A, Keever-Taylor CA, et al. Bispecific anti-CD20, anti-CD19 CAR T cells for relapsed B cell malignancies: a phase 1 dose escalation and expansion trial. Nat Med. 2020;26:1569-75.

47. Tong C, Zhang Y, Liu Y, Ji X, Zhang W, Guo Y, et al. Optimized tandem CD19/CD20 CAR-engineered T cells in refractory/relapsed B-cell lymphoma. Blood. 2020;136:1632-44.

48. Amrolia PJ, Wynn R, Hough RE, Vora A, Bonney D, Veys P, et al. Phase Study of AUTO3, a bicistronic chimeric antigen receptor (CAR) T-cell therapy targeting CD19 and CD22, in pediatric patients with relapsed/ refractory B-cell acute lymphoblastic leukemia ( $r / r$ B-ALL): Amelia Study. Blood. 2019:134:2620-2620.

49. Osborne W, Marzolini M, Tholouli E, Ramakrishnan A, Bachier CR, McSweeney PA, et al. Phase I Alexander study of AUTO3, the first CD19/22 dual targeting CART cell therapy, with pembrolizumab in patients with relapsed/refractory $(r / r)$ DLBCL. J Clin Oncol Wolters Kluwer. 2020:38:8001-8001.

50. Pegram HJ, Lee JC, Hayman EG, Imperato GH, Tedder TF, Sadelain $\mathrm{M}$, et al. Tumor-targeted T cells modified to secrete IL-12 eradicate systemic tumors without need for prior conditioning. Blood. 2012;119:4133-41.

51. Avanzi MP, Yeku O, Li X, Wijewarnasuriya DP, van Leeuwen DG, Cheung $\mathrm{K}$, et al. Engineered tumor-targeted T cells mediate enhanced antitumor efficacy both directly and through activation of the endogenous immune system. Cell Rep. 2018;23:2130-41.

52. Kuhn NF, Purdon TJ, van Leeuwen DG, Lopez AV, Curran KJ, Daniyan $A F$, et al. CD40 ligand-modified chimeric antigen receptor $T$ cells enhance antitumor function by eliciting an endogenous antitumor response. Cancer Cell. 2019;35:473-488.e6.

53. Lai J, Mardiana S, House IG, Sek K, Henderson MA, Giuffrida L, et al. Adoptive cellular therapy with T cells expressing the dendritic cell growth factor Flt3L drives epitope spreading and antitumor immunity. Nat Immunol. 2020;21:914-26.

54. Stephan MT, Ponomarev V, Brentjens RJ, Chang AH, Dobrenkov KV Heller G, et al. T cell-encoded CD80 and 4-1BBL induce auto- and transcostimulation, resulting in potent tumor rejection. Nat Med Nature Publishing Group. 2007;13:1440-9.

55. DeSelm C, Palomba ML, Yahalom J, Hamieh M, Eyquem J, Rajasekhar VK, et al. Low-dose radiation conditioning enables CAR T cells to mitigate antigen escape. Mol Ther J Am Soc Gene Ther. 2018;26:2542-52.

56. Laurent SA, Hoffmann FS, Kuhn P-H, Cheng Q, Chu Y, Schmidt-Supprian $\mathrm{M}$, et al. $\mathrm{Y}$-Secretase directly sheds the survival receptor BCMA from plasma cells. Nat Commun. 2015;6:7333.

57. Pont MJ, Hill T, Cole GO, Abbott JJ, Kelliher J, Salter Al, et al. $\mathrm{g}$-Secretase inhibition increases efficacy of BCMA-specific chimeric antigen receptor $T$ cells in multiple myeloma. :13.

58. Ruella M, Barrett DM, Shestova O, Perazzelli J, Posey AD, Hong SJ, et al. A cellular antidote to specifically deplete anti-CD19 chimeric antigen receptor-positive cells. Blood. 2020;135:505-9.

59. Green MR, Monti S, Rodig SJ, Juszczynski P, Currie T, O'Donnell E, et al. Integrative analysis reveals selective 9p24.1 amplification, increased PD-1 ligand expression, and further induction via JAK2 in nodular sclerosing Hodgkin lymphoma and primary mediastinal large B-cell lymphoma. Blood. 2010;116:3268-77.

60. Ansell SM, Minnema MC, Johnson P, Timmerman JM, Armand P, Shipp MA, et al. Nivolumab for relapsed/refractory diffuse large B-cell lymphoma in patients ineligible for or having failed autologous transplantation: a single-arm, Phase II Study. J Clin Oncol Off J Am Soc Clin Oncol. 2019;37:481-9.

61. Armand P, Rodig S, Melnichenko V, Thieblemont C, Bouabdallah K, Tumyan $\mathrm{G}$, et al. Pembrolizumab in relapsed or refractory primary mediastinal large B-cell lymphoma. J Clin Oncol Off J Am Soc Clin Oncol. 2019:37:3291-9.

62. Feucht J, Kayser S, Gorodezki D, Hamieh M, Döring M, Blaeschke F, et al. T-cell responses against CD19+ pediatric acute lymphoblastic leukemia mediated by bispecific T-cell engager (BiTE) are regulated contrarily by PD-L1 and CD80/CD86 on leukemic blasts. Oncotarget. 2016;7:76902-19.

63. Park SH, You E, Park C-J, Cho Y-U, Jang S, Im H-J, et al. Increased expression of immune checkpoint programmed cell death protein-1 (PD-1) on T cell subsets of bone marrow aspirates in patients with B-Lymphoblastic leukemia, especially in relapse and at diagnosis. Cytometry B Clin Cytom. 2020;98:336-47.

64. Perez A, Navale L, Rossi JM, Shen Y, Jiang Y, Sherman M, et al. Pharmacodynamic profile and clinical response in patients with B-cell malignancies of anti-CD19 CAR T-cell therapy. Blood. 2015;126:2042-2042.

65. Neelapu SS, Locke FL, Bartlett NL, Lekakis LJ, Miklos DB, Jacobsen ED, et al. Long-term follow-up ZUMA-1: a pivotal trial of axicabtagene ciloleucel (Axi-Cel; KTE-C19) in patients with refractory aggressive non-Hodgkin Lymphoma (NHL). Blood. 2017:130:578-578.

66. Chong EA, Svoboda J, Dwivedy Nasta S, Landsburg DJ, Winchell $\mathrm{N}$, Napier $\mathrm{E}$, et al. Sequential anti-CD19 directed chimeric antigen receptor modified T-cell therapy (CART19) and PD-1 blockade with pembrolizumab in patients with relapsed or refractory B-cell nonHodgkin Lymphomas. Blood. 2018;132:4198-4198.

67. Hirayama AV, Gauthier J, Hay KA, Sheih A, Cherian S, Chen X, et al. Efficacy and toxicity of JCAR014 in combination with durvalumab for the treatment of patients with relapsed/refractory aggressive B-cell non-Hodgkin Lymphoma. Blood. 2018;132:1680-1680.

68. Jacobson CA, Locke FL, Miklos DB, Herrera AF, Westin JR, Lee J, et al. End of Phase 1 results from zuma-6: axicabtagene ciloleucel (Axi-Cel) in combination with atezolizumab for the treatment of patients with refractory diffuse large B cell lymphoma. Biol Blood Marrow Transplant. 2019;25:S173.

69. Rafiq S, Yeku OO, Jackson HJ, Purdon TJ, van Leeuwen DG, Drakes DJ, et al. Targeted delivery of a PD-1-blocking scFv by CAR-T cells enhances anti-tumor efficacy in vivo. Nat Biotechnol. 2018;36:847-56. 
70. Rupp LJ, Schumann K, Roybal KT, Gate RE, Ye CJ, Lim WA, et al. CRISPR/ Cas9-mediated PD-1 disruption enhances anti-tumor efficacy of human chimeric antigen receptor T cells. Sci Rep. 2017;7:737.

71. Stadtmauer EA, Fraietta JA, Davis MM, Cohen AD, Weber KL, Lancaster $E$, et al. CRISPR-engineered T cells in patients with refractory cancer. Science. 2020;367.

72. Ren J, Liu X, Fang C, Jiang S, June $\mathrm{CH}$, Zhao Y. Multiplex genome editing to generate universal CAR T cells resistant to PD1 inhibition. Clin Cancer Res Off J Am Assoc Cancer Res. 2017;23:2255-66.

73. Cherkassky L, Morello A, Villena-Vargas J, Feng Y, Dimitrov DS, Jones DR, et al. Human CART cells with cell-intrinsic PD-1 checkpoint blockade resist tumor-mediated inhibition. J Clin Invest. 2016;126:3130-44.

74. Liu H, Lei W, Zhang C, Yang C, Wei J, Guo Q, et al. CD19-specific CART cells that express a PD-1/CD28 chimeric switch-receptor are effective in patients with PD-L1-positive B-cell lymphoma. Clin Cancer Res Off J Am Assoc Cancer Res. 2021;27:473-84.

75. Bullens DMA, Rafiq K, Charitidou L, Peng X, Kasran A, Warmerdam PAM, et al. Effects of co-stimulation by CD58 on human T cell cytokine production: a selective cytokine pattern with induction of high IL-10 production. Int Immunol. 2001;13:181-91.

76. Leitner J, Herndler-Brandstetter D, Zlabinger GJ, Grubeck-Loebenstein B, Steinberger P. CD58/CD2 is the primary costimulatory pathway in human CD28-CD8+ T cells. J Immunol Am Assoc Immunol. 2015;195:477-87.

77. Majzner R. CD58 Aberrations Limit Durable Responses to CD19 CAR in Large B Cell Lymphoma Patients Treated with Axicabtagene Ciloleucel but Can be Overcome through Novel CAR Engineering. ASH; 2020 [cited 2021 Jul 3]. Available from: https://ash.confex.com/ash/2020/ webprogram/Paper139605.html

78. Lemoine J, Ruella M, Houot R. Overcoming intrinsic resistance of cancer cells to CAR T-cell killing. Clin Cancer Res Off J Am Assoc Cancer Res. 2021

79. Singh N, Lee YG, Shestova O, Ravikumar P, Hayer KE, Hong SJ, et al. Impaired death receptor signaling in leukemia causes antigen-independent resistance by inducing CAR T-cell dysfunction. Cancer Discov. 2020;10:552-67.

80. Dufva O, Koski J, Maliniemi P, lanevski A, Klievink J, Leitner J, et al. Integrated drug profiling and CRISPR screening identify essential pathways for CAR T-cell cytotoxicity. Blood. 2020;135:597-609.

81. Müller M, Wilder S, Bannasch D, Israeli D, Lehlbach K, Li-Weber M, et al. p53 activates the CD95 (APO-1/Fas) gene in response to DNA damage by anticancer drugs. J Exp Med. 1998;188:2033-45.

82. Fandy TE, Shankar S, Ross DD, Sausville E, Srivastava RK. Interactive effects of HDAC inhibitors and TRAIL on apoptosis are associated with changes in mitochondrial functions and expressions of cell cycle regulatory genes in multiple myeloma. Neoplasia N Y N. 2005;7:646-57.

83. He Q, Huang Y, Sheikh MS. Proteasome inhibitor MG132 upregulates death receptor 5 and cooperates with Apo2L/TRAIL to induce apoptosis in Bax-proficient and -deficient cells. Oncogene. 2004;23:2554-8.

84. Huang Y, He Q, Hillman MJ, Rong R, Sheikh MS. Sulindac sulfide-induced apoptosis involves death receptor 5 and the caspase 8-dependent pathway in human colon and prostate cancer cells. Cancer Res. 2001:61:6918-24

85. Torres-Collado AX, Jazirehi AR. Overcoming resistance of human non-Hodgkin's Lymphoma to CD19-CAR CTL therapy by celecoxib and histone deacetylase inhibitors. Cancers. 2018;10:200.

86. Song JH, Kandasamy K, Kraft AS. ABT-737 Induces Expression of the Death Receptor 5 and Sensitizes Human Cancer Cells to TRAIL-induced Apoptosis. J Biol Chem Am Soc Biochem Mol Biol. 2008;283:25003-13.

87. Yang M, Wang L, Ni M, Neuber B, Wang S, Gong W, et al. Pre-sensitization of malignant $B$ cells through venetoclax significantly improves the cytotoxic efficacy of CD19.CAR-T Cells. Front Immunol. 2020;11:608167.

88. Smith MR. Rituximab (monoclonal anti-CD20 antibody): mechanisms of action and resistance. Oncogene. 2003;22:7359-68.

89. Ryland GL, Barraclough A, Fong CY, Fleming S, Bajel A, Hofmann O, et al. Inotuzumab ozogamicin resistance associated with a novel CD22 truncating mutation in a case of B-acute lymphoblastic leukaemia. Br J Haematol. 2020;191:123-6.

90. Hergott CB, Kim AS, Wadleigh M, Lindsley RC. Resistance to inotuzumab ozogamicin in a B-ALL patient with TET2 and DNMT3A mutations and myeloid lineage switch. Blood. 2018;132:2818.

91. Peranzoni E, Lemoine J, Vimeux L, Feuillet V, Barrin S, Kantari-Mimoun $C$, et al. Macrophages impede CD8 T cells from reaching tumor cells and limit the efficacy of anti-PD-1 treatment. Proc Natl Acad Sci. 2018;115:E4041-50.

92. Salmon H, Franciszkiewicz K, Damotte D, Dieu-Nosjean M-C, Validire P, Trautmann A, et al. Matrix architecture defines the preferential localization and migration of T cells into the stroma of human lung tumors. J Clin Invest. 2012;122:899-910.

93. Boulch M, Cazaux M, Loe-Mie Y, Thibaut R, Corre B, Lemaitre F, et al. A cross-talk between CART cell subsets and the tumor microenvironment is essential for sustained cytotoxic activity. Sci Immunol. 2021;6:eabd4344

94. Shah BD, Ghobadi A, Oluwole OO, Logan AC, Boissel N, Cassaday RD, et al. KTE-X19 for relapsed or refractory adult B-cell acute lymphoblastic leukaemia: phase 2 results of the single-arm, open-label, multicentre ZUMA-3 study. The Lancet. 2021;S0140673621012228.

95. Chen J, López-Moyado IF, Seo H, Lio C-WJ, Hempleman LJ, Sekiya T, et al. NR4A transcription factors limit CAR T cell function in solid tumours. Nature. 2019;567:530-4.

96. Shen C, Zhang Z, Zhang Y. Chimeric antigen receptor T cell exhaustion during treatment for hematological malignancies. BioMed Res Int. 2020;2020:8765028.

97. Liu G, Rui W, Zheng H, Huang D, Yu F, Zhang Y, et al. CXCR2-modified CAR-T cells have enhanced trafficking ability that improves treatment of hepatocellular carcinoma. Eur J Immunol. 2020;50:712-24.

98. Guo Y, Wang Y, Han W. chimeric antigen receptor-modified T cells for solid tumors: challenges and prospects. J Immunol Res. 2016;2016:3850839.

99. Kershaw MH, Westwood JA, Parker LL, Wang G, Eshhar Z, Mavroukakis $\mathrm{SA}$, et al. A Phase I study on adoptive immunotherapy using genemodified T cells for ovarian cancer. Clin Cancer Res. 2006;12:6106-15.

100. Knochelmann HM, Smith AS, Dwyer CJ, Wyatt MM, Mehrotra S, Paulos CM. CART cells in solid tumors: blueprints for building effective therapies. Front Immunol. 2018;9:1740.

101. Donnadieu E, Dupré L, Pinho LG, Cotta-de-Almeida V. Surmounting the obstacles that impede effective CART cell trafficking to solid tumors. J Leukoc Biol. 2020:108:1067-79.

102. Kosti P, Larios-Martinez Kl, Maher J, Arnold JN. Generation of hypoxiasensing chimeric antigen receptor T cells. STAR Protoc. 2021;2:100723.

103. Liao Q, He H, Mao Y, Ding X, Zhang X, Xu J. Engineering T cells with hypoxia-inducible chimeric antigen receptor (HiCAR) for selective tumor killing. Biomark Res. 2020;8:56

104. Juillerat A, Marechal A, Filhol JM, Valogne Y, Valton J, Duclert A, et al. An oxygen sensitive self-decision making engineered CAR T-cell. Sci Rep. 2017;7:39833

105. Martinez M, Moon EK. CART cells for solid tumors: new strategies for finding, infiltrating, and surviving in the tumor microenvironment. Front Immunol. 2019:10:128.

106. Yan Z-X, Li L, Wang W, OuYang B-S, Cheng S, Wang L, et al. Clinical efficacy and tumor microenvironment influence in a dose-escalation study of anti-CD19 chimeric antigen receptor T cells in refractory B-cell non-Hodgkin's Lymphoma. Clin Cancer Res Off J Am Assoc Cancer Res. 2019:25:6995-7003.

\section{Publisher's Note}

Springer Nature remains neutral with regard to jurisdictional claims in published maps and institutional affiliations. 